Internat. J. Math. \& Math. Sci.

Vol. 24, No. 3 (2000) 193-201

S0161171200002441

(C) Hindawi Publishing Corp.

\title{
ON ALMOST PRECONTINUOUS FUNCTIONS
}

\section{SAEID JAFARI and TAKASHI NOIRI}

(Received 19 December 1997 and in revised form 28 September 1998)

\begin{abstract}
Nasef and Noiri (1997) introduced and investigated the class of almost precontinuous functions. In this paper, we further investigate some properties of these functions.

Keywords and phrases. Preopen, preclosed, preboundary, strongly compact, almost precontinuous, strongly prenormal.
\end{abstract}

2000 Mathematics Subject Classification. Primary 54C10; Secondary 54C08.

1. Introduction. Singal and Singal [24] introduced the notion of almost continuity. Feeble continuity was introduced by Maheshwari et al. [8]. As a generalization of almost continuity and feeble continuity, Maheshwari et al. [7] introduced the notion of almost feeble continuity. Nasef and Noiri [12] introduced a new class of functions called almost precontinuous functions. They showed that almost precontinuity is a generalization of each of almost feeble continuity and almost $\alpha$-continuity [17].

The purpose of this paper is to investigate some more properties of almost precontinuous functions. It turns out that almost precontinuity is stronger than almost weak continuity introduced by Jankovič [5].

2. Preliminaries. Throughout this paper, $(X, \tau)$ and $(Y, \sigma)$ (or $X$ and $Y$ ) are always topological spaces. A set $A$ in a space $X$ is called preopen [11] (respectively, semiopen [6] and $\alpha$-open [13]) if $A \subset \stackrel{\circ}{\bar{A}}$ (respectively, $A \subset \overline{A^{\circ}}$ and $A \subset \overline{A^{\circ}}{ }^{\circ}$ ). The complement of a preopen set is called preclosed.

The intersection of all preclosed sets containing a subset $A$ is called the preclosure [2] of $A$ and is denoted by $\operatorname{Pcl}(A)$. The preinterior of $A$ is the union of all preopen sets of $X$ contained in $A$. The family of all preopen sets of $X$ will be denoted by $\operatorname{PO}(X)$. For a point $x$ of $X$, we put $\mathrm{PO}(X, x)=\{U \mid x \in U \in \mathrm{PO}(X)\}$. A set $A$ is called regular open (respectively, regular closed) if $A=\stackrel{\circ}{A}$ (respectively, $A=\bar{A}^{\circ}$ ).

DefinITION 2.1. A function $f: X \rightarrow Y$ is called almost continuous [24] (in the sense of Singal) at $x \in X$ if for every open set $V$ in $Y$ containing $f(x)$, there is an open set $U$ in $X$ containing $x$ such that $f(U) \subset \bar{V}^{\circ}$. If $f$ is almost continuous at every point of $X$, then it is called almost continuous.

DEFINITION 2.2. A function $f: X \rightarrow Y$ is called almost weakly continuous [5] (briefly a.w.c.) if $f^{-1}(V) \subset{\overline{f^{-1}(\bar{V})}}^{\circ}$ for every open set $V$ of $Y$.

REMARK 2.3. In [20, Theorem 3.1] Popa and Noiri have defined the following pointwise description of almost weak continuity: a function $f: X \rightarrow Y$ is a.w.c. if and 
only if for each point $x \in X$ and every open set $V$ in $Y$ containing $f(x)$, there exists $U \in \mathrm{PO}(X, x)$ such that $f(U) \subset \bar{V}$. The referee has given a global description as follows: a function $f: X \rightarrow Y$ is a.w.c. if and only if for each open set $V$ of $Y$, there exists $U \in \mathrm{PO}(X)$ such that $f^{-1}(V) \subset U \subset f^{-1}(\bar{V})$.

DEFINITION 2.4. A function $f: X \rightarrow Y$ is called almost precontinuous [12] (briefly a.p.c.) at $x \in X$ if for each regular open set $V \subset Y$ containing $f(x)$, there exists $U \in$ $\mathrm{PO}(X, x)$ such that $f(U) \subset V$. If $f$ is almost precontinuous at every point of $X$, then it is called almost precontinuous.

DEFINITION 2.5. A function $f: X \rightarrow Y$ is said to be weakly $\alpha$-continuous [16] (briefly w. $\alpha$.c.) if for each $x \in X$ and each open set $V \subset Y$ containing $f(x)$, there exists an $\alpha$ open set $U$ containing $x$ such that $f(U) \subset \bar{V}$.

DEFINITION 2.6. A function $f: X \rightarrow Y$ is said to be precontinuous [11] if for every open set $V$ of $Y$, the inverse image of $V$ is preopen in $X$.

REMARK 2.7. Between almost precontinuity and precontinuity, we have the following relationship: a function $f: X \rightarrow Y$ is a.p.c. if and only if $f: X \rightarrow Y_{s}$ is precontinuous, where $Y_{s}$ denotes the semi-regularization of $Y$.

REMARK 2.8. It easily follows from [20, Theorem 3.1] that precontinuity implies almost precontinuity and almost precontinuity implies almost weak continuity. However, the converses are not true as the following examples show.

EXAMPLE 2.9. Let $X=\{a, b, c\}, \tau=\{X, \varnothing,\{a\},\{c\},\{a, c\}\}$ and $\sigma=\{X, \varnothing,\{a\},\{b\}$, $\{a, b\},\{b, c\}\}$. Define a function $f:(X, \tau) \rightarrow(X, \sigma)$ as follows: $f(a)=f(b)=b$ and $f(c)=c$. Then $f$ is an almost continuous and hence a.p.c. function which is not precontinuous. Because, there exists $\{b\} \in \sigma$ such that $f^{-1}(\{b\}) \notin \operatorname{PO}(X, \boldsymbol{\tau})$.

EXAMPLE 2.10. Let $X=\{a, b, c, d\}$ and $\tau=\{X, \varnothing,\{b\},\{c\},\{a, b\},\{b, c\},\{a, b, c\}$, $\{b, c, d\}\}$. Define a function $f:(X, \tau) \rightarrow(X, \tau)$ as follows: $f(a)=c, f(b)=d, f(c)=$ $b$, and $f(d)=a$. Then $f$ is a.w.c. However, $f$ is not a.p.c. because there exists a regular open set $\{c\}$ of $(X, \tau)$ such that $f^{-1}(\{c\}) \notin \mathrm{PO}(X, \tau)$.

Recall that a filter base $\mathscr{F}$ is called $\delta$-convergent [25] (respectively, $p$-convergent [4]) to a point $x$ in $X$ if for any open set $U$ containing $x$ (respectively, any $U \in \operatorname{PO}(X, x)$ ), there exists $B \in \mathscr{F}$ such that $B \subset \bar{U}^{\circ}$ (respectively, $B \subset U$ ).

3. Some properties. In [9], Mashhour et al. introduced the following notion.

DefinITION 3.1. A function $f: X \rightarrow Y$ is called $M$-preopen if the image of each preopen set is preopen.

We have the following result.

THEOREM 3.2. If $f: X \rightarrow Y$ is $M$-preopen a.w.c., then $f$ is a.p.c.

Proof. Suppose that $x \in X$ and $V$ is any open set containing $f(x)$. Since $f$ is a.w.c., then there exists $U \in \mathrm{PO}(X, x)$ such that $f(U) \subset \bar{V}$ [20, Theorem 3.1]. Since $f$ is 
$M$-preopen, $f(U)$ is preopen in $Y$ and hence $f(U) \subset \overline{f(U)}^{\circ} \subset \overline{\bar{V}}^{\circ}=\bar{V}^{\circ}$. It follows that $f(U) \subset \bar{V}^{\circ}$. Hence $f$ is a.p.c.

Recall that a space $X$ is called submaximal if every dense subset of $X$ is open in $X$. It is shown in [22, Theorem 4] that a space $X$ is submaximal if and only if every preopen set of $X$ is open in $X$.

THEOREM 3.3. If a function $f: X \rightarrow Y$ is a.p.c., then for each point $x \in X$ and each filter base $\mathscr{F}$ in $X p$-converging to $x$, the filter base $f(\mathscr{F})$ is $\delta$-convergent to $f(x)$. If $X$ is submaximal, then the converse also holds.

Proof. Suppose that $x$ belongs to $X$ and $\mathscr{F}$ is any filter base in $X p$-converging to $x$. By the almost precontinuity of $f$, for any regular open set $V$ in $Y$ containing $f(x)$, there exists $U \in \mathrm{PO}(X, x)$ such that $f(U) \subset V$. But $\mathscr{F}$ is $p$-convergent to $x$ in $X$, then there exists $B \in \mathscr{F}$ such that $B \subset U$. It follows that $f(B) \subset V$. This means that $f(\mathscr{F})$ is $\delta$-convergent to $f(x)$.

Now suppose that $X$ is submaximal. Let $x$ be a point in $X$ and $V$ any regular open set containing $f(x)$. Since $X$ is submaximal, every preopen set of $X$ is open [22, Theorem 4]. If we set $\mathscr{F}=\mathrm{PO}(X, x)$, then $\mathscr{F}$ will be a filter base which $p$-converges to $x$. So there exists $U$ in $\mathscr{F}$ such that $f(U) \subset V$. This completes the proof.

The following corollary is suggested by the referee.

COROLLARY 3.4. Let $X$ be a submaximal space. Then a function $f: X \rightarrow Y$ is a.p.c. if and only if $f: X \rightarrow Y_{s}$ is continuous.

DEFINITION 3.5. A space $X$ is called pre- $T_{2}$ [18] if for every pair of distinct points $x$ and $y$ in $X$, there exist preopen sets $U$ and $V$ containing $x$ and $y$, respectively, such that $U \cap V=\varnothing$.

THEOREM 3.6. If $f: X \rightarrow Y$ is an a.p.c. injection and $Y$ is Hausdorff, then $X$ is $p r e-T_{2}$.

Proof. Since $f: X \rightarrow Y$ is a.p.c. injective, $f: X \rightarrow Y_{s}$ is a precontinuous injection and $Y_{s}$ is Hausdorff. Let $x$ and $y$ be any distinct points of $X$. Since $f$ is injective, $f(x) \neq f(y)$ and hence there exist disjoint open sets $V$ and $W$ of $Y_{s}$ such that $f(x) \in V$ and $f(y) \in W$. Therefore, we obtain $f^{-1}(V) \in \mathrm{PO}(X, x), f^{-1}(W) \in \mathrm{PO}(X, y)$, and $f^{-1}(V) \cap f^{-1}(W)=\varnothing$. This shows that $X$ is pre- $T_{2}$.

Recall that a space $X$ is called a door space if every subset of $X$ is either open or closed. Reilly and Vamanamurthy proved the following result in [22, Theorem 2].

LEMMA 3.7. If $X$ is a door space, then every preopen set in $X$ is open.

THEOREM 3.8. Let $f, g: X \rightarrow Y$ be functions, $Y$ Hausdorff and $X$ a door space. If $f$ and $g$ are a.p.c. functions, then the set $E=\{x \in X \mid f(x)=g(x)\}$ is closed in $X$.

Proof. Let $x \in X-E$. It follows that $f(x) \neq g(x)$. Since $Y$ is Hausdorff, then there exist open sets $V_{1}$ and $V_{2}$ in $Y$ such that $f(x) \in V_{1}, g(x) \in V_{2}$, and $V_{1} \cap V_{2}=\varnothing$. Since $V_{1}$ and $V_{2}$ are disjoint, we obtain $\bar{V}_{1}^{\circ} \cap \bar{V}_{2}^{\circ}=\varnothing$. Since $f$ and $g$ are a.p.c., there exist preopen sets $U_{1}$ and $U_{2}$ in $X$ containing $x$ such that $f\left(U_{1}\right) \subset \bar{V}_{1}^{\circ}$ and $g\left(U_{2}\right) \subset \bar{V}_{2}^{\circ}$. Put 
$U=U_{1} \cap U_{2}$, so, by Lemma 3.7, $U$ is an open set in $X$ containing $x$. Thus we have $f(U) \cap g(U)=\varnothing$. It follows that $x \notin \bar{E}$. Hence $\bar{E} \subset E$ and $E$ is closed in $X$.

LEMMA 3.9 (Popa and Noiri [20]). If $A$ is an $\alpha$-open set of a space $X$ and $B \in \mathrm{PO}(X)$, then $A \cap B \in \mathrm{PO}(X)$.

THEOREM 3.10. Let $f, g: X \rightarrow Y$ be functions and $Y$ Hausdorff. If $f$ is w.x.c. and $g$ is a.p.c., then the set $E=\{x \in X \mid f(x)=g(x)\}$ is preclosed in $X$.

Proof. Suppose that $x \notin E$. Then $f(x) \neq g(x)$. Since $Y$ is Hausdorff, there exist open sets $V$ and $W$ of $Y$ such that $f(x) \in V, g(x) \in W$, and $V \cap W=\varnothing$; hence $\bar{V} \cap \bar{W}^{\circ}=$ $\varnothing$. Since $f$ is w. $\alpha$.c., there exists an $\alpha$-open set $U$ containing $x$ such that $f(U) \subset \bar{V}$. Since $g$ is a.p.c., there exists $G \in \operatorname{PO}(X, x)$ such that $g(G) \subset \bar{W}^{\circ}$. Put $O=U \cap G$, then $O \in \operatorname{PO}(X, x)$ by Lemma 3.9 and $O \cap E=\varnothing$. Therefore, we obtain $x \notin \operatorname{Pcl}(E)$. This shows that $E$ is preclosed in $X$.

COROLlary 3.11 (Popa [19]). Let $f, g: X \rightarrow Y$ be functions and $Y$ Hausdorff. If $f$ is continuous and $g$ is precontinuous, then the set $E=\{x \in X \mid f(x)=g(x)\}$ is preclosed in $X$.

THEOREM 3.12. Let $f: X_{1} \rightarrow Y$ and $g: X_{2} \rightarrow Y$ be two a.p.c. functions. If $Y$ is a Hausdorff space, then the set $\left\{\left(x_{1} \times x_{2}\right) \in X_{1} \times X_{2} \mid f\left(x_{1}\right)=g\left(x_{2}\right)\right\}$ is preclosed in $X_{1} \times X_{2}$.

Proof. Let $\left(x_{1}, x_{2}\right) \notin E$. Then $f\left(x_{1}\right) \neq g\left(x_{2}\right)$. Since $Y$ is Hausdorff, there exist disjoint open neighborhoods $V$ and $W$ of $f\left(x_{1}\right)$ and $g\left(x_{2}\right)$, respectively. Since $V$ and $W$ are disjoint, we have $\bar{V}^{\circ} \cap \bar{W}^{\circ}=\varnothing$. Since $f$ and $g$ are a.p.c., there exist $U \in \operatorname{PO}\left(X_{1}, x_{1}\right)$ and $G \in \mathrm{PO}\left(X_{2}, x_{2}\right)$ such that $f(U) \subset \bar{V}^{\circ}$ and $g(G) \subset \bar{W}^{\circ}$, respectively. Put $O=U \times$ $G$, then $\left(x_{1}, x_{2}\right) \in O, O$ is preopen in $X_{1} \times X_{2}$ and $O \cap E=\varnothing$. Therefore, we obtain $\left(x_{1}, x_{2}\right) \in \operatorname{Pcl}(E)$. This shows that $E$ is preclosed in $X_{1} \times X_{2}$.

COROLLARY 3.13. If $Y$ is Hausdorff and $f: X \rightarrow Y$ is an a.p.c. function, then the set $E=\{(x, y) \mid f(x)=f(y)\}$ is preclosed in $X \times X$.

Proof. By setting $X=X_{1}=X_{2}$ and $g=f$ in Theorem 3.12, the result follows.

COROLLARY 3.14 (Mashhour et al. [11]). If $f: X \rightarrow Y$ is a precontinuous function and $Y$ is Hausdorff, then the set $\{(x, y) \mid f(x)=f(y)\}$ is preclosed in $X \times X$.

Corollary 3.15 (Popa [19]). Let $f: X_{1} \rightarrow Y$ and $g: X_{2} \rightarrow Y$ be two precontinuous functions. If $Y$ is a Hausdorff space, then the set $\{(x, y) \mid f(x)=g(y)\}$ is preclosed in $X_{1} \times X_{2}$.

We introduce the following concept.

DEFINITION 3.16. For a function $f: X \rightarrow Y$, the graph $G(f)=\{(x, f(x)) \mid x \in X\}$ is called strongly almost preclosed if for each $(x, y) \in X \times Y-G(f)$, there exist $U \in$ $\mathrm{PO}(X, x)$ and a regular open set $V$ containing $y$ such that $(U \times V) \cap G(f)=\varnothing$.

LEMMA 3.17. A function $f: X \rightarrow Y$ has the strongly almost preclosed graph if and only if for each $x \in X$ and $y \in Y$ such that $f(x) \neq y$, there exist $U \in \mathrm{PO}(X, x)$ and $a$ regular open set $V$ containing $y$ such that $f(U) \cap V=\varnothing$. 
Proof. It is an immediate consequence of the above definition.

THEOREM 3.18. If $f: X \rightarrow Y$ is a.w.c. and $Y$ is Hausdorff, then $G(f)$ is strongly almost preclosed.

Proof. Suppose that $(x, y)$ is any point of $X \times Y-G(f)$. Then $y \neq f(x)$. But $Y$ is Hausdorff and hence there exist open sets $G_{1}$ and $G_{2}$ in $Y$ such that $y \in G_{1}, f(x) \in G_{2}$, and $G_{1} \cap G_{2}=\varnothing$. Since $G_{1}$ and $G_{2}$ are disjoint, we obtain $\bar{G}_{1}^{\circ} \cap \bar{G}_{2}=\varnothing$. And since $f$ is a.w.c., then there exists $U \in \mathrm{PO}(X, x)$ such that $f(U) \subset \bar{G}_{2}$. Hence, $f(U) \cap \bar{G}_{1}^{\circ}=\varnothing$. It follows from Lemma 3.17 that $G(f)$ is strongly almost preclosed.

Recall that a subset $A$ of a space $X$ is said to be strongly compact relative to $X$ [9] (respectively, $N$-closed relative to $X[1]$ ) if every cover of $A$ by preopen (respectively, regular open) sets of $X$ has a finite subcover.

DEFINITION 3.19. A space $X$ is called strongly compact [10] (respectively, nearly compact [23]) if every preopen (respectively, regular open) cover of $X$ has a finite subcover.

THEOREM 3.20. If $f: X \rightarrow Y$ is a.p.c. and $K$ is a strongly compact relative to $X$, then $f(K)$ is $N$-closed relative to $Y$.

PROof. Let $\left\{G_{\alpha} \mid \alpha \in A\right\}$ be any cover of $f(K)$ by regular open sets of $Y$. Then, $\left\{f^{-1}\left(G_{\alpha}\right) \mid \alpha \in A\right\}$ is a cover of $K$ by preopen sets of $X$ [12, Theorem 3.1]. Since $K$ is strongly compact relative to $X$, there exists a finite subset $A_{\circ}$ of $A$ such that $K \subset \cup\left\{f^{-1}\left(G_{\alpha}\right) \mid \alpha \in A_{\circ}\right\}$. Therefore, we obtain $f(K) \subset \cup\left\{G_{\alpha} \mid \alpha \in A_{\circ}\right\}$. This shows that $f(K)$ is $N$-closed relative to $Y$.

COROLLARY 3.21. If $f: X \rightarrow Y$ is an a.p.c. surjection and $X$ is strongly compact, then $Y$ is nearly compact.

DeFinITION 3.22. A function $f: X \rightarrow Y$ is said to be $\delta$-continuous [14] if for each $x \in X$ and each open set $V$ of $Y$ containing $f(x)$, there exists an open set $U$ in $X$ containing $x$ such that $f\left(\bar{U}^{\circ}\right) \subset \bar{V}^{\circ}$.

THEOREM 3.23. If $f: X \rightarrow Y$ is a.p.c. and $g: Y \rightarrow Z$ is $\delta$-continuous, then $g \circ f: X \rightarrow Z$ is a.p.c.

Proof. The proof is obvious and is omitted.

THEOREM 3.24. If $f: X \rightarrow Y$ is an M-preopen surjection and $g: Y \rightarrow Z$ is a function such that $g \circ f: X \rightarrow Z$ is a.p.c., then $g$ is a.p.c.

Proof. Let $y \in Y$ and $x \in X$ such that $f(x)=y$. Let $G$ be a regular open set containing $(g \circ f)(x)$. Then there exists $U \in \mathrm{PO}(X, x)$ such that $g(f(U)) \subset G$. Since $f$ is $M$-preopen, $f(U) \in \mathrm{PO}(Y, y)$ such that $g(f(U)) \subset G$. This shows that $g$ is a.p.c. at $y$.

THEOREM 3.25. If $f: X \rightarrow Y$ is a.p.c. and $A$ is a semi-open set of $X$, then the restriction $f \mid A: A \rightarrow Y$ is a.p.c.

Proof. Let $V$ be any regular open set of $Y$. Since $f$ is a.p.c., the inverse image of $V$ is preopen in $X\left[12\right.$, Theorem 3.1] and $(f \mid A)^{-1}(V)=A \cap f^{-1}(V)$. Since $A$ is 
semi-open in $X$, it follows from [11, Lemma 2.1] that $A \cap f^{-1}(V) \in \operatorname{PO}(A)$. Therefore, $f \mid A$ is a.p.c.

REMARK 3.26. It should be noted that every restriction of an a.p.c. function is not necessarily a.p.c. In [15, proof of Theorem 6.2.5], it is pointed out that there is a precontinuous function whose restriction to a not semi-open set is not even a.w.c. It might also be noted that neither is almost precontinuity for a function $f: X \rightarrow Y$ preserved by restriction of the codomain to $f(X)$. The following example is due to referee.

EXAMPLE 3.27. Let $f: \mathbb{Q} \rightarrow \mathbb{R}$ be the inclusion map of the rationals into the reals. Let the domain have the usual subspace topology and let the nonempty open sets in the codomain have the form $P \cup A$, where $P=\mathbb{R}-\mathbb{Q}$ is the set of irrationals and where $A \subseteq \mathbb{Q}$. Then $\mathbb{R}_{S}$ is indiscrete so that $f$ is a.p.c. Yet, $f(\mathbb{Q})$ is a discrete subspace of $\mathbb{R}$ so that $f: \mathbb{Q} \rightarrow f(\mathbb{Q})$ is not a.p.c. since not every subset of the domain space is preopen.

THEOREM 3.28. Let $f: X \rightarrow Y$ be a function and $x \in X$. If there exists $U \in \mathrm{PO}(X, x)$ such that the restriction of $f$ to $U$ is a.p.c. at $x$, then $f$ is a.p.c. at $x$.

Proof. Suppose that $V_{2}$ is any regular open set containing $f(x)$. Since $f \mid U$ is a.p.c. at $x$, there exists $V_{1} \in \mathrm{PO}(U, x)$ such that $f\left(V_{1}\right)=(f \mid U)\left(V_{1}\right) \subset V_{2}$. Since $U \in$ $\mathrm{PO}(X, x)$, it follows from [11, Lemma 2.2] that $V_{1} \in \mathrm{PO}(X, x)$. This shows clearly that $f$ is a.p.c. at $x$.

DEFINITION 3.29. Let $A \subset X$. The preboundary $\operatorname{pFr}(A)$ of $A$ is defined by $\operatorname{pFr}(A)=$ $\operatorname{Pcl}(A) \cap \operatorname{Pcl}(X-A)$.

THEOREM 3.30. The set of all points $x$ of $X$ at which $f: X \rightarrow Y$ is not a.p.c. is identical with the union of the preboundaries of the inverse images of regular open subsets of $Y$ containing $f(x)$.

Proof. If $f$ is not a.p.c. at $x \in X$, then there exists a regular open set $V$ containing $f(x)$ such that for every $U \in \operatorname{PO}(X, x), f(U) \cap(Y-V) \neq \varnothing$. This means that for every $U \in \mathrm{PO}(X, x)$, we must have $U \cap\left(X-f^{-1}(V)\right) \neq \varnothing$. Hence, it follows from [2, Lemma 2.2] that $x \in \operatorname{Pcl}\left(X-f^{-1}(V)\right)$. But $x \in f^{-1}(V)$ and hence $x \in \operatorname{Pcl}\left(f^{-1}(V)\right)$. This means that $x$ belongs to the preboundary of $f^{-1}(V)$. Suppose that $x$ belongs to the preboundary of $f^{-1}\left(V_{1}\right)$ for some regular open subset $V_{1}$ of $Y$ such that $f(x) \in V_{1}$. Suppose that $f$ is a.p.c. at $x$. Then there exists $U \in \operatorname{PO}(X, x)$ such that $f(U) \subset V_{1}$. Then, we have: $x \in U \subset f^{-1}(f(U)) \subset f^{-1}\left(V_{1}\right)$. This shows that $x$ is a preinterior point of $f^{-1}\left(V_{1}\right)$. Therefore, we have $x \notin \operatorname{Pcl}\left(X-f^{-1}\left(V_{1}\right)\right)$ and $x \notin \operatorname{pFr}\left(f^{-1}\left(V_{1}\right)\right)$. But this is a contradiction. This means that $f$ is not a.p.c.

Recall that a subset $A$ of a space $X$ is said to be $H$-set [25] or quasi $H$-closed relative to $X$ [21] if for every cover $\left\{U_{i} \mid i \in I\right\}$ of $A$ by open sets of $X$, there exists a finite subset $I_{0}$ of $I$ such that $A \subset \cup\left\{\bar{U}_{i} \mid i \in I_{0}\right\}$.

THEOREM 3.31. If $f: X \rightarrow Y$ is a.w.c. and $K$ is strongly compact relative to $X$, then $f(K)$ is quasi $H$-closed relative to $Y$.

Proof. The proof is similar to the one of Theorem 3.20. 
Recall that a function $f: X \rightarrow Y$ is called $r$-preopen [3] if the image of a preopen set in $X$ is open in $Y$.

THEOREM 3.32. Let $f: X \rightarrow Y$ be an a.w.c. bijection. If $X$ is strongly compact and $Y$ is Hausdorff, then $f$ is $r$-preopen.

Proof. Suppose that $U$ is a preopen subset of $X$. Then $X-U$ is preclosed subset of the strongly compact space $X$. This means that $X-U$ is strongly compact relative to $X$. By Theorem 3.31, $f(X-U)$ is quasi $H$-closed relative to $Y$. Since $f$ is bijective, we have $f(X-U)=Y-f(U)$, where $Y-f(U)$ is quasi $H$-closed relative to $Y$. Since $Y$ is Hausdorff, therefore $Y-f(U)$ is closed in $Y$. Hence $f(U)$ is open in $Y$.

COROLLARY 3.33. Let $f: X \rightarrow Y$ be an a.p.c. bijection. If $X$ is strongly compact and $Y$ is Hausdorff, then $f$ is $r$-preopen.

Proof. Since every a.p.c. function is a.w.c., hence the proof follows from Theorem 3.32 .

DeFinition 3.34. Let $E$ and $F$ be any two subsets of $X$. $E$ and $F$ are called strongly $p$-separated if there exist disjoint preopen sets $U$ and $V$ such that $E \subset U$ and $F \subset V$.

DEFINITION 3.35. A function $f: X \rightarrow Y$ is said to be strongly preclosed [18] if the image of a preclosed set in $X$ is preclosed in $Y$.

DEFINITION 3.36. A space $X$ is called strongly prenormal [18] if for disjoint preclosed subsets $E$ and $F$ of $X$, there exist disjoint preopen sets $U$ and $V$ such that $E \subset U$ and $F \subset V$.

THEOREM 3.37. If $f$ is an a.p.c., strongly preclosed function of strongly pre-normal space $X$ onto a space $Y$, then any two disjoint regular closed subsets of $Y$ can be strongly p-separated.

Proof. Let $F$ and $D$ be two disjoint regular closed subsets of $Y$. Then $f^{-1}(F)$ and $f^{-1}(D)$ are disjoint, preclosed subsets of the strongly prenormal space $X$ and therefore there exist preopen sets $U$ and $W$ such that $U \cap W=\varnothing, f^{-1}(F) \subset U$, and $f^{-1}(D) \subset W$. Suppose that

$$
P_{1}=\left\{y \mid f^{-1}(y) \subset U\right\}, \quad P_{2}=\left\{y \mid f^{-1}(y) \subset W\right\} .
$$

Since $f$ is strongly preclosed, then $P_{1}$ and $P_{2}$ are preopen sets. Then we have

$$
F \subset P_{1}, \quad D \subset P_{2}, \quad P_{1} \cap P_{2}=\varnothing .
$$

Now we obtain the following results whose proofs are omitted since they are straightforward.

Recall that a space $X$ is said to be extremally disconnected if the closure of each open set of $X$ is open in $X$.

THEOREM 3.38. If $f: X \rightarrow Y$ is a.w.c. and $Y$ is extremally disconnected, then $f$ is a.p.c. 
ACKNOWLEDGEMENT. The authors are very grateful to the referee for his careful work and suggestions that improved this paper very much.

\section{REFERENCES}

[1] D. Carnahan, Locally nearly-compact spaces, Boll. Un. Mat. Ital. (4) 6 (1972), 146-153. MR 47\#9546. Zbl 257.54020.

[2] N. El-Deeb, I. A. Hasanein, A. S. Mashhour, and T. Noiri, On p-regular spaces, Bull. Math. Soc. Sci. Math. R. S. Roumanie (N.S.) 27(75) (1983), no. 4, 311-315. MR 85d:54018. Zbl 524.54016.

[3] S. Jafari, On rarely precontinuous functions, Far East J. Math. Sci., to appear.

[4] S. Jafari and T. Noiri, Functions with preclosed graphs, Univ. Bacău. Stud. Cerc. St. Ser. Mat. 8 (1998), 53-56.

[5] D. S. Jankovič, $\theta$-regular spaces, Int. J. Math. Math. Sci. 8 (1985), no. 3, 615-619. MR 87h:54030. Zbl 577.54012.

[6] N. Levine, Semi-open sets and semi-continuity in topological spaces, Amer. Math. Monthly 70 (1963), 36-41. MR 29\#4025. Zbl 113.16304.

[7] S. N. Maheshwari, G. I. Chae, and P. C. Jain, Almost feebly continuous functions, Ulsan Inst. Tech. Rep. 13 (1982), no. 1, 195-197. MR 83h:54014. Zbl 482.54007.

[8] S. N. Maheshwari and P. C. Jain, Some new mappings, Mathematica (Cluj) 24(47) (1982), no. 1-2, 53-55. MR 84k:54009. Zbl 513.54008.

[9] A. S. Mashhour, M. E. Abd El-Monsef, and I. A. Hasanein, On pretopological spaces, Bull. Math. Soc. Sci. Math. R. S. Roumanie (N.S.) 28(76) (1984), no. 1, 39-45. MR 85k:54017. Zbl 532.54002.

[10] A. S. Mashhour, M. E. Abd El-Monsef, I. A. Hasanein, and T. Noiri, Strongly compact spaces, Delta J. Sci. 8 (1984), no. 1, 30-46.

[11] A. S. Mashhour, I. A. Hasanein, and S. N. El-Deeb, A note on semi-continuity and precontinuity, Indian J. Pure Appl. Math. 13 (1982), no. 10, 1119-1123. Zbl 499.54009.

[12] A. A. Nasef and T. Noiri, Some weak forms of almost continuity, Acta Math. Hungar. 74 (1997), no. 3, 211-219. CMP 1440 246. Zbl 924.54017.

[13] O. Njästad, On some classes of nearly open sets, Pacific J. Math. 15 (1965), 961-970. MR 33\#3245. Zbl 137.41903.

[14] T. Noiri, On $\delta$-continuous functions, J. Korean Math. Soc. 16 (1979/80), no. 2, 161-166. MR 82b:54020. Zbl 435.54010.

[15] _ Properties of some weak forms of continuity, Int. J. Math. Math. Sci. 10 (1987), no. 1, 97-111. MR 88a:54028. Zbl 617.54008.

[16] W Weakly $\alpha$-continuous functions, Int. J. Math. Math. Sci. 10 (1987), no. 3, 483-490. MR 88f:54016. Zbl 638.54012.

[17] _ Almost $\alpha$-continuous functions, Kyungpook Math. J. 28 (1988), no. 1, 71-77. MR 90a:54032. Zbl 675.54011.

[18] T. M. J. Nour, Contributions to the Theory of Bitopological Spaces, Ph.D. thesis, University of Delhi, 1989.

[19] V. Popa, Properties of H-almost continuous functions, Bull. Math. Soc. Sci. Math. R. S. Roumanie (N.S.) 31(79) (1987), no. 2, 163-168. MR 88k:54028. Zbl 618.54013.

[20] V. Popa and T. Noiri, Almost weakly continuous functions, Demonstratio Math. 25 (1992), no. 1-2, 241-251. MR 93f:54020. Zbl 789.54014.

[21] J. Porter and J. Thomas, On H-closed and minimal Hausdorff spaces, Trans. Amer. Math. Soc. 138 (1969), 159-170. MR 38\#6544. Zbl 175.49501.

[22] I. L. Reilly and M. K. Vamanamurthy, On some questions concerning preopen sets, Kyungpook Math. J. 30 (1990), no. 1, 87-93. MR 91h:54021. Zbl 718.54004.

[23] M. K. Singal and A. Mathur, On nearly-compact spaces, Boll. Un. Mat. Ital. (4) 2 (1969), 702-710. MR 41\#2628. Zbl 188.28005.

[24] M. K. Singal and A. R. Singal, Almost-continuous mappings, Yokohama Math. J. 16 (1968), 63-73. MR 41\#6182. Zbl 191.20802. 
[25] N. V. Veličko, H-closed topological spaces, Amer. Math. Soc. Transl. (2) 78 (1968), 103-118.

SAEID JAFARI: DEPARTMENT OF MATHEMATICS AND Physics, ROSKILDE UNIVERSITY, P.O. BOX 2604000 ROSKILDE, DENMARK

E-mail address: jafari@post12 . te1e.dk

TAKashi Noiri: Department of Mathematics, Yatsushiro College of Technology, YATSUSHIRO-SHI, KUMAMOTO-KEN, 866, JAPAN

E-mail address: noi ri@as.yatsushi ro-nct.ac.jp 


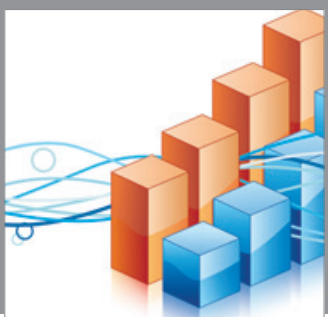

Advances in

Operations Research

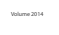

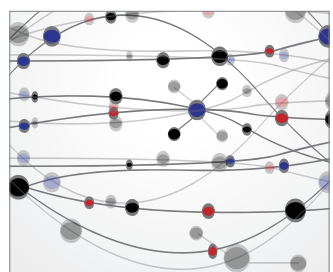

\section{The Scientific} World Journal
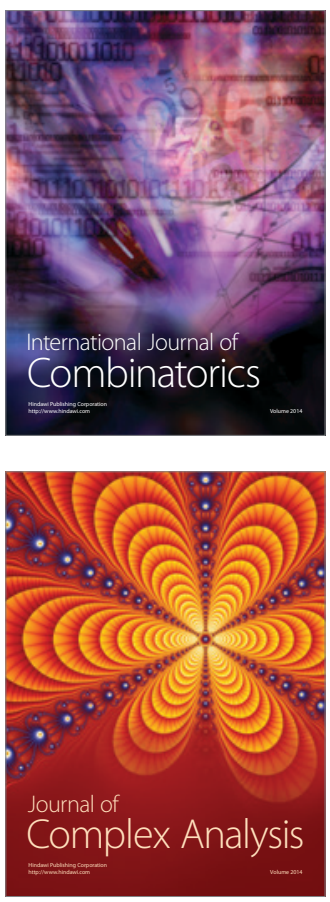

International Journal of

Mathematics and

Mathematical

Sciences
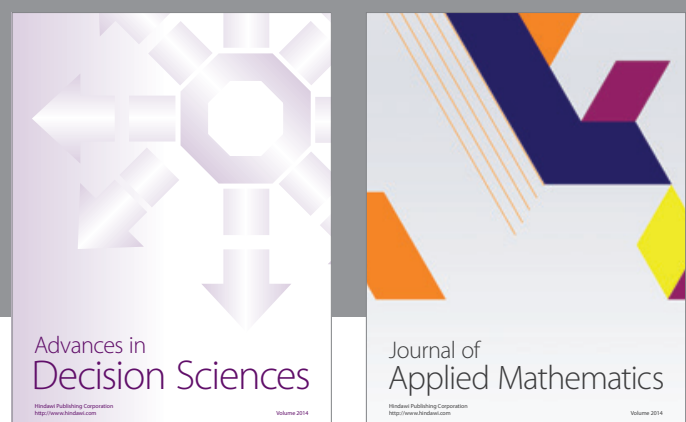

Journal of

Applied Mathematics
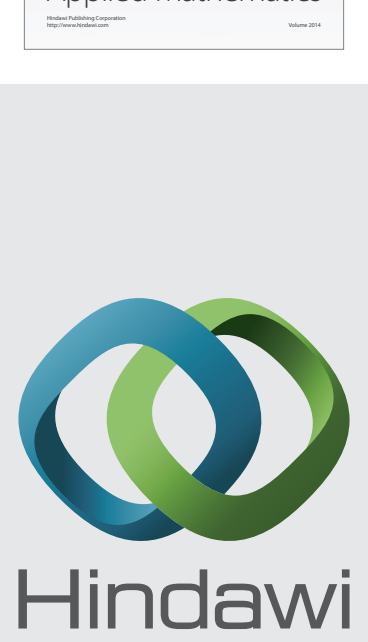

Submit your manuscripts at http://www.hindawi.com
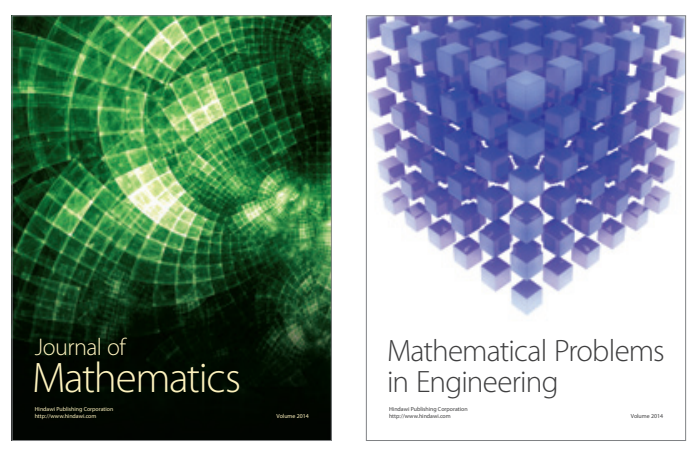

Mathematical Problems in Engineering
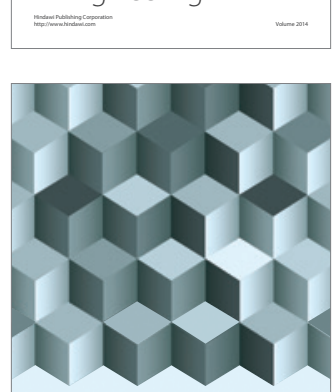

Journal of

Function Spaces
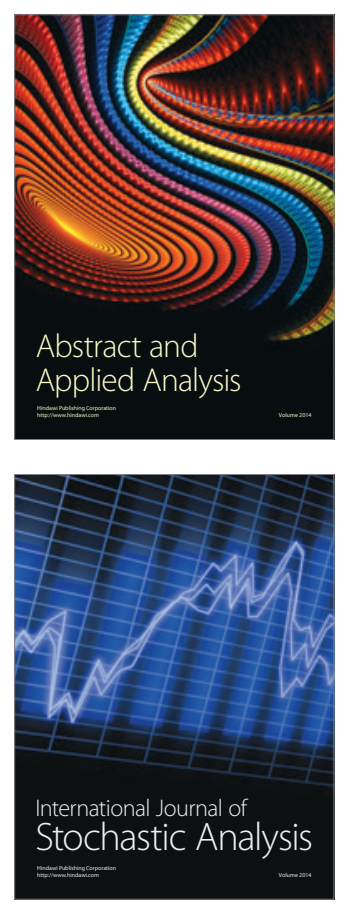

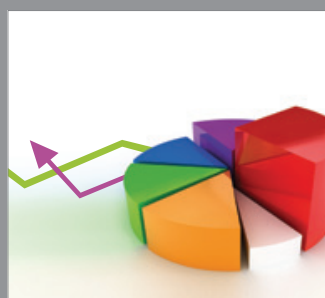

ournal of

Probability and Statistics

Promensencen
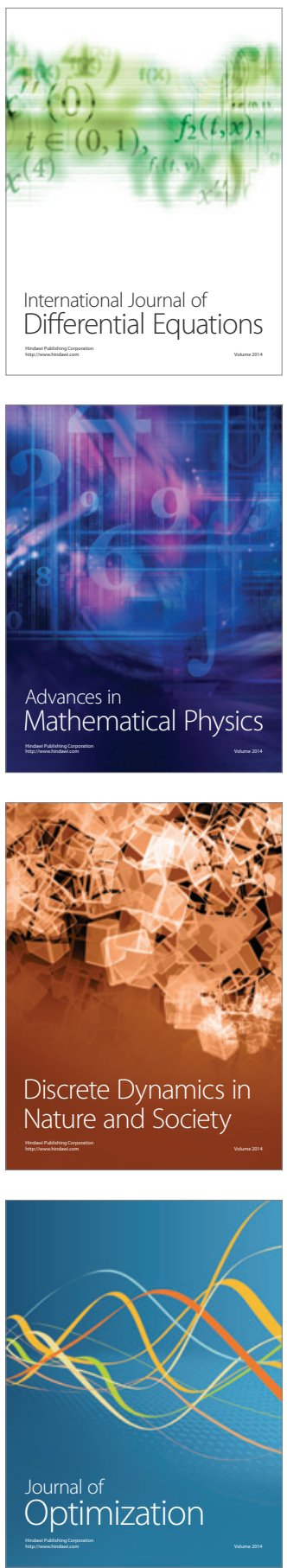